\title{
Temporal Behavior of Radiation-Pressure-Induced Vibrations of an Optical Microcavity Phonon Mode
}

\author{
Tal Carmon, Hossein Rokhsari, Lan Yang, Tobias J. Kippenberg, and Kerry J. Vahala* \\ California Institute of Technology, 1200 E. California Boulevard, Pasadena, California 91125 USA
}

(Received 22 November 2004; published 10 June 2005)

\begin{abstract}
We analyze experimentally and theoretically mechanical oscillation within an optical cavity stimulated by the pressure of circulating optical radiation. The resulting radio frequency cavity vibrations (phonon mode) cause modulation of the incident, continuous-wave (cw) input pump beam. Furthermore, with increasing cw pump power, an evolution from sinusoidal modulation to random oscillations is observed in the pump power coupled from the resonator. The temporal evolution with pump power is studied, and agreement was found with theory. In addition to applications in quantum optomechanics, the present work suggests that radiation-pressure-induced effects can establish a practical limit for the miniaturization of optical silica microcavities.
\end{abstract}

DOI: 10.1103/PhysRevLett.94.223902

PACS numbers: 42.79.Jq, 42.55.Sa

Optical cavities with walls pushed by radiation pressure (RP) have similar properties to cavities containing a Kerr medium [1] including hysteretic wavelength response caused by RP [2]. In particular, both RP and the Kerr effect induce a coupling between optical path length and light intensity, one through cavity strain and the other through the index of refraction. It is therefore not surprising that RP has been proposed as an alternative path for observation of quantum effects such as squeezing [1] and creation of EPR entangled pairs of photons [3]. Moreover, the nature of RP to act on a macroscopic object suggests that RP is a promising mechanism for entangling macroscopic mechanical oscillators [4], creating a Schrödinger cat state of a macroscopic mirror [5], teleportation of a macroscopic mechanical resonator state [6] and more.

We present here a microscale device consisting only of an ultra-high- $Q(\mathrm{UH} Q)$ toroid cavity and a cw pump wave. The pressure of circulating radiation induces a mechanical flex of the cavity structure; this motion, in return, takes the optical cavity out of resonance with the $\mathrm{cw}$ pump wave, thereby lowering RP. Upon restoration of the mechanical flex, the process resumes, leading to a periodic motion of the cavity as well as the circulating power. It should be emphasized that this oscillation is regenerative, exhibiting classic threshold behavior and requiring no external temporal modulation of the pump wave. Recently, transmission oscillations were reported, which were shown to occur exactly at the mechanical eigenfrequencies of the cavity structure [7]. Here, we explore and explain both experimentally and theoretically the system's temporal behavior. In particular, it is shown that the output intensity of the system evolves from sinusoidal to a train of decaying peaks and finally to random oscillations as input $\mathrm{cw}$ pump power increases.

It is also shown here that when the size of a silica cavity is small, oscillations can occur well before other nonlinear effects as well as before lasing due to the intentional doping of rare earths into the microcavities. Namely, we demonstrate that these regenerative vibrations can occur at pump powers lower than for Kerr parametric oscillation [8], as well as for Raman [9] and erbium lasing [10] (when the cavity was made to be small enough). This miniaturization limit can be extended by adding a mechanical damper (or displacement limiter) to the cavity structure. Yet, the fundamental nature of circulating light to apply pressure, and the general nature of structural stiffness to reduce with miniaturization [11], should be considered.

We note that RP as a nonlinear mechanism is very different from another important nonlinearity: the thermal nonlinearity [12]. While the thermal nonlinearity is driven by absorption and hence has a dissipative character, the RP nonlinearity is reactive and strongly depends on the optical quality factor. The RP nonlinearity increases with the mechanical and optical quality factor, because more acoustical and optical energy stays in the cavity. Scaling arguments show that RP will increase quadratically with miniaturization for a fixed $Q$ factor, while the thermal nonlinearity increases less rapidly. The consequence of these differences is that RP dominates when the cavity optical and mechanical quality factor is high and structure size is small.

The experimental system (Fig. 1) consists of an $\mathrm{UH} Q$ toroid [13], fabricated from silica on a silicon wafer. Optical coupling of the $\mathrm{cw}$ pump laser to (and from) the microresonator is performed via a tapered fiber $[14,15]$. It should be noted that the authors have observed the same vibrational effect in spherical cavities and hence it is not exclusive to a specific cavity structure. Oscillations in transmitted pump power appear above a threshold power level and evolve with increasing power from sinusoidal [Fig. 2(a), left] to a train of decaying ripples [Fig. 2(d)2(g)] and finally to a seemingly chaotic behavior [Fig. 2(h)]. The oscillations are observed to continue as long as $\mathrm{cw}$ pump power is maintained.

In modeling the ring cavity, we note that each circulating photon changes its propagation direction twice every revo- 


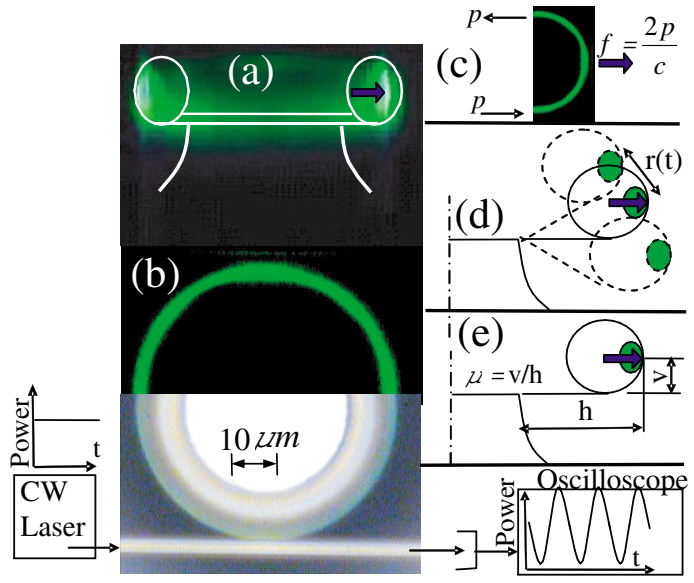

FIG. 1 (color online). Experimental setup. Side view (a) and top view (b) of a $\mathrm{UH} Q$ toroidal microcavity. A $\mathrm{cw}$ pump laser is coupled by way of a fiber taper and builds a circulating intensity that applies pressure on the microcavity walls. In (c) and (e) circulating power induces a radial force, which causes modulation of the cantilever as illustrated in (d) and the pump wave as illustrated in (b). Green luminescence in panels (a) and (b) is the result of erbium up-conversion (from 1.5 to $0.5 \mu \mathrm{m}$ ) and is used here only to illustrate the location of the optical mode. Note that the optical mode (green) is higher than the "cantilever beam" [(a), (e)] holding the microtoroid.

lution [Fig. 1(c)]. Therefore, a photon transfers 4 times its linear momentum to the cavity's walls every time it completes a round trip. If the cavity is not infinitely rigid, the walls will deform in response to the resulting pressure [Fig. 1(d)]. In particular, the position of the cavity ring, $r(t)$ [Fig. 1(d)], will evolve according to the following equation of motion for a mechanical oscillator:

$$
m \ddot{r}(t)+b \dot{r}(t)+k r(t)=\mu f(t)=\mu 2 \pi|A(t)|^{2} n / c,
$$

where $m$ is the ring mass, $b$ is the mechanical dissipation, $k$ is a spring constant, $f$ is the horizontal force applied by action of RP, and $\mu$ is the force efficiency defined in Fig. 1(e) that gives the conversion of horizontal to lateral force through the moment arm of the disk. Based on the photo in Fig. 1(a), $\mu$ was estimated here to be $1 / 3 . A(t)$ is the slowly varying field amplitude normalized so that $|A(t)|^{2}$ is the circulating optical power, and $c / n$ is the velocity of light in the cavity. The calculated radiation force on the right hand side is the consequence of total momentum conservation [Fig. 1(c)]. As the optical resonance shifts with structure expansion, the wavelength difference (between the input beam and the moving-cavity resonance) changes as

$$
\Delta \omega(t)=\Delta \omega_{0}-\omega \mu r(t) 2 \pi n /\left(\lambda_{0} N\right),
$$

where $\Delta \omega_{0}$ is the preset offset between cavity resonance (when no light is input) and the input pump beam at frequency $\omega$. In addition, $N=2 \pi R n / \lambda_{0}$ is the number of optical resonance wavelengths along the cavity circumference, and $\mu r$ is the deviation in the radius of the optical mode. The circulating optical field amplitude (normalized such that $|A(t)|^{2}$ is optical power) evolves according to the dynamical equation [16]

$$
\dot{A}(t)+A(t)\left[\frac{\alpha c}{n}-i \Delta \omega(t)\right]=i B \sqrt{\frac{\alpha c}{n \tau_{0}}},
$$

where $\alpha$ is the optical loss in the cavity (in $\mathrm{cm}^{-1}$ ), $B$ is the input pump field (normalized such that $|B|^{2}$ is optical power), and $\tau_{0}=n L / c$ is the circulation time for a photon traveling inside the cavity where $L=2 \pi R$ is approximately equal to the circumference of the cavity.

Applying the predictor-corrector Adams' method on Eqs. (1)-(3) reveals the system dynamical behavior $[A(t)$,

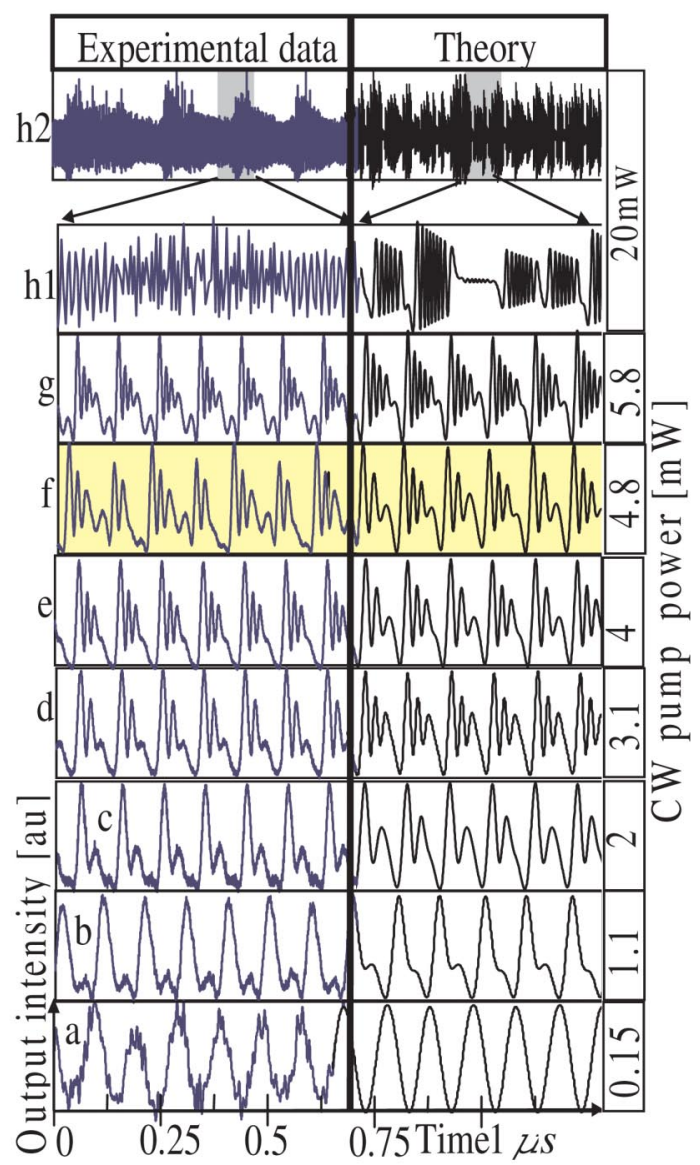

FIG. 2 (color online). Transmitted pump power versus time for various values of $\mathrm{cw}$ input power. Left: experimental results. Right: calculated variation in optical power. Here, the opticalmode radius is $R=29 \mu \mathrm{m}$ and the refractive index is $n=1.46$. The ring's mass was calculated (from measured ring size) to be $5 \times 10^{-11} \mathrm{~kg}$; the mechanical dissipation was inferred from measured subthreshold acoustical bandwidth versus pump power to be $b=1.4 \times 10^{-6} \mathrm{~kg} / \mathrm{s}$ and the mechanical oscillation frequency was measured to be $5.4 \mathrm{MHz}$. The pump wavelength is fixed at a wavelength that is 0.55 FWHM less than the cavity optical resonance ( $\lambda_{0}=1461 \mathrm{~nm}$ ) and the optical quality factor was measured to be $Q_{o}=5 \times 10^{7}$ [except in panel (h), where $Q_{o}=2 \times 10^{8}$ and $\lambda_{0}=1550 \mathrm{~nm}$ ). Panel (h1) is also presented in panel (h2) with a time scale 8 times longer. 
$r(t)]$. Oscillations typically evolve to their full scale (starting from mechanical rest) within 5 time constants of the mechanical cavity $(10 \mathrm{~m} / \mathrm{b})$. Having $A(t)$, the calculated oscillating output power $\quad\left(\mid\left(1-\tau_{0} \alpha c / 2 n\right) B+\right.$ $\left.i \sqrt{\tau_{0} \alpha c / n} A(t)\right|^{2}$ as explained in Ref. [16]) is presented in Fig. 2 (right hand side). The system response for low input pump power resembles a laser line modulated by a sinusoidally moving Lorentzian line shape [17]. In particular, for small oscillation amplitudes a nearly linear amplitude relation exists between mechanical motion and power modulation, because of the offset in pump frequency from the microcavity resonance. As the Lorentzian deviation becomes larger, the system response becomes nonlinear.

As pumping further increases, output power transforms into a train of decaying peaks [Fig. 2(c)-2(g)]. This decaying-peaks behavior is the result of interference between the stationary pump light and the light emitted from the cavity upon discharging. Output intensity, here, reflects the fact that light discharged from the cavity is decaying exponentially with time and is frequency shifted due to cavity vibrations. We note that, while the system motion is predictable for small enough pump levels [Fig. 2(a)-2(g)], it becomes sensitive to infinitesimal noise in the initial condition when a specific threshold in the pump power is exceeded [Fig. 2(h)]. In this aspect, this system resembles the chaotic behavior of the famous, damped-driven pendulum.

A single measurement of output power [Fig. 3(a)] will now be analyzed in detail. Note first that calculated output power [Fig. 3(b)] is in good agreement with experimental results [Fig. 3(a)]. Cavity position $r(t)$ [Fig. 3(c)] exhibits a flex of a few $\AA$, which corresponds to a resonance shift of a few cavity bandwidths. The cavity resonance crosses the pump laser wavelength when wall velocity $\left[\partial_{t} r(t)\right]$ is about $0.9 \mathrm{~mm} / \mathrm{s}$ [Fig. 3(d)]. The cavity is then charged to a circulating power $\left(|A(t)|^{2}\right)$ that approaches $50 \mathrm{~W}$ [Fig. 3(e)] causing a centrifugal radiation force $(f)$ that approaches $1.4 \mu \mathrm{N}$. Upon cavity expansion, the power $\left[f(t) \partial_{t} \mu r(t)\right]$ applied by radiation on the moving structure approaches $0.2 \mathrm{nW}$ [Fig. 3(f)]. The resulting transference of energy is manifested as a red Doppler shift in the circulating photons. Upon the cavity shrinking, power flows back to the optical mode from the mechanical potential energy; the circulating photons then experience a blue Doppler shift. A significant point is the need for the pump wavelength to be lower than the microcavity resonance in order to induce oscillation. Because of this offset, the pump wave spends a little more time to one side of the microcavity resonant frequency [Fig. 3(c)]. This asymmetry in resonance motion explains why energy $\left[\int_{0}^{t} f\left(t^{\prime}\right) \partial_{t^{\prime}} \mu r\left(t^{\prime}\right) d t^{\prime}\right]$ flows on average from the optical mode to the acoustical mode [Fig. 3(g)]. In the example, for each acoustical cycle, $9 \times 10^{-18} \mathrm{~J}$ of energy are given to the optical mode upon shrinking, but then $10 \times 10^{-18} \mathrm{~J}$ are taken from the optical mode upon expansion
[Fig. 3(g)]. This energy difference drives the mechanical vibrations. The sign of the energy flow will be reversed if the pump-microcavity frequency offset changes sign. The asymmetry in resonance motion also explains the surprising alternating number of peaks in Figs. 3(a) and 3(b) (4, 3, $4,3, \ldots)$.

Going to Fourier space, the calculated and measured (using a Fabry-Perot spectrometer having a $1 \mathrm{MHz}$ linewidth) field spectrum are given in Fig. 3(h); note that this spectrum is asymmetric. The calculated field spectrum

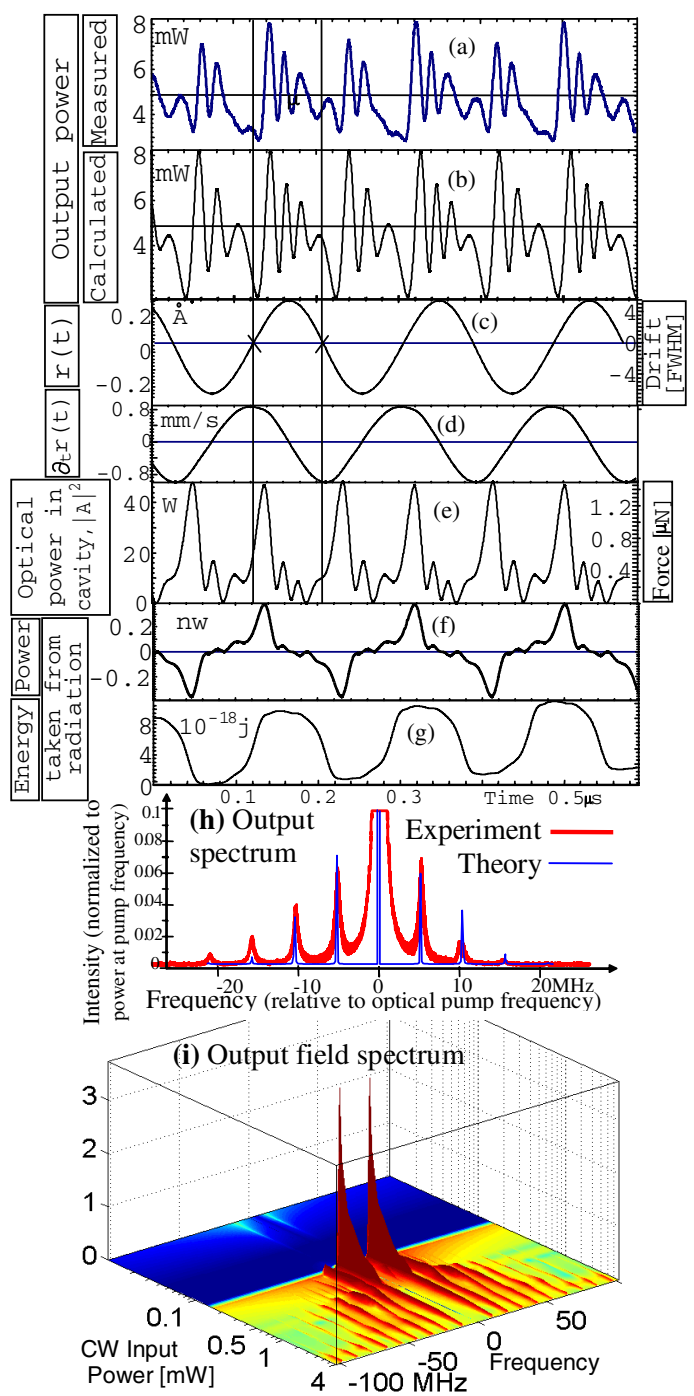

FIG. 3 (color online). Plots versus time of (a) measured and (b) calculated transmitted optical power, (c) cavity's walls displacement and (d) velocity, (e) optical power circulating in cavity (left ordinate) which is proportional to the total radial force (right ordinate) applied by radiation on the cavity walls, (f) power flow to phonon mode and (g) energy transferred to phonon mode from radiation. (h) Measured and calculated spectrum of the transmitted optical power; (i) Calculated spectrum of the transmitted field (normalized to transmitted pump which is not shown) as a function of input pump power. Parameters in this figure are as in Fig. 2; pump power is $4.8 \mathrm{~mW}$ in (a)-(f) and $2 \mathrm{~mW}$ in (h). 


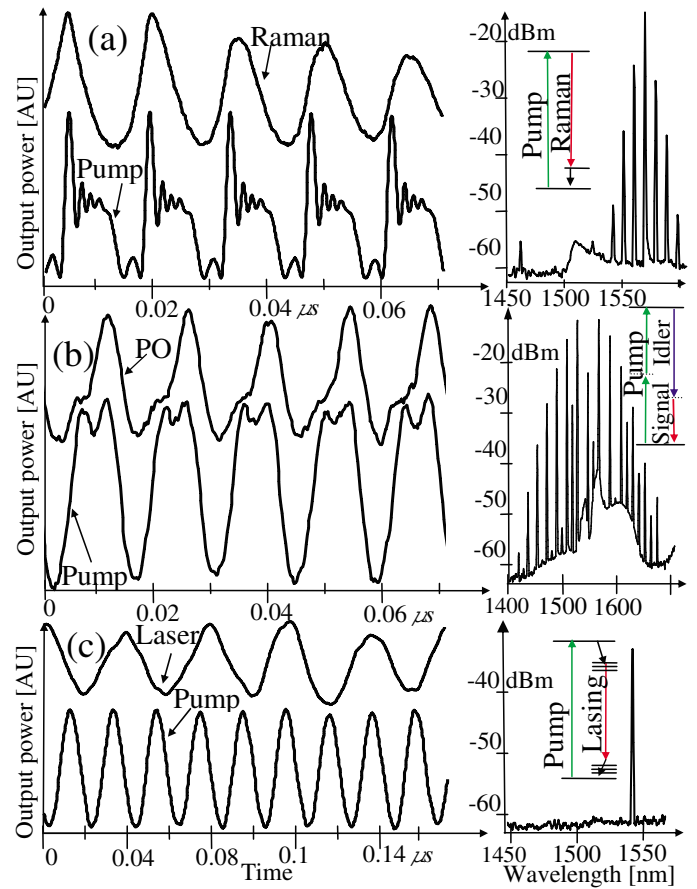

FIG. 4 (color online). Coexistence of RP-induced vibrations with (a) Raman lasing, (b) Kerr parametric oscillations, and (c) erbium lasing. On the left: temporal behavior of scattered pump and emitted signal. Right: signal spectrum. In these experiments RP-induced vibrations threshold was lower than the signal threshold. Pump wavelength is at 1462, 1555, and $1458 \mathrm{~nm}$; pump power is 4,20 , and $1 \mathrm{~mW}$, respectively.

[Fig. 3(i)] versus pump power reveals a clear oscillation threshold at input power of about $0.15 \mathrm{~mW}$ [18]. As input power increases, the system response is large enough to induce a nonlinear amplitude modulation of the incident pump field. This manifests itself though the appearance of higher-order sidebands in the field spectrum (generally referred to as Stokes and anti-Stokes frequencies).

In general, when different optical nonlinearities are in competition, the nonlinearity with the highest gain wins and governs. RP-induced vibrations were, however, observed (Fig. 4) to coexist with Kerr parametric oscillations [8], Raman [9], and erbium lasing [10] ("other" processes). In the specific experiments presented in Fig. 4 the threshold for RP nonlinearities was lower than the threshold for the other processes. Consequently, other processes appear only when the cavity is already vibrating. The other processes were observed in spite of the fact that cavity vibrations induce drifts of few cavity FWHM on time scales smaller than $1 \mu$ s. Raman lasing [Fig. 4(a)] and Kerr parametric oscillations [Fig. 4(b)] were observed to exhibit power modulation synchronous with cavity vibrations [19]. It is also interesting that the RP-induced nonlinearity, which originates from vibrations of a macroscopic structure, coexists with Raman lasing that originates from molecular vibrations.

To conclude, radiation-pressure-induced stimulation of regenerative (radio frequency) mechanical oscillations of a silica microcavity have been demonstrated experimentally and analyzed theoretically. The vibrational mode and the optical mode act as (parametrically) coupled oscillators despite their very different frequencies $\left(\sim 10^{7}\right.$ against $\sim 10^{14} \mathrm{~Hz}$ ). The oscillations in output optical power grow from a continuous-wave input beam and evolve with increasing pump power from sinusoids, to a train of decaying peaks (sometimes, with alternating peak number), and finally to random oscillation. Scaling arguments suggest that RP should be considered in other micro-resonant optical devices where high intensities are confined to a small volume. As miniaturization can improve key features of many optical devices, there is much effort aimed at bringing devices to the micron and nanosize levels. We hence expect that more and more optical devices will encounter RP related effects, whether as a limiting floor in miniaturization or as a useful new optomechanical nonlinearity.

*Corresponding author.

Email address: vahala@caltech.edu

Electronic address: http://vahala.caltech.edu/

[1] L. Hilico et al., Appl. Phys. B 55, 202 (1992).

[2] A. Dorsel, et al., Phys. Rev. Lett. 51, 1550 (1983).

[3] V. Giovannetti, S. Mancini, and P. Tombesi, Europhys. Lett. 54, 559 (2001).

[4] S. Mancini et al., Phys. Rev. Lett. 88, 120401 (2002).

[5] W. Marshall et al., Phys. Rev. Lett. 91, 130401 (2003).

[6] S. Mancini, D. Vitali, and P. Tombesi, Phys. Rev. Lett. 90, 137901 (2003).

[7] H. Rokhsari, T. J. Kippenberg, T. Carmon, and K. J. Vahala, (to be published).

[8] T. J. Kippenberg, S. M. Spillane, and K. J. Vahala, Phys. Rev. Lett. 93, 083904 (2004).

[9] S. M. Spillane, T. J. Kippenberg, and K. J. Vahala, Nature (London) 415, 621 (2002).

[10] S. L. Mccall et al., Appl. Phys. Lett. 60, 289 (1992).

[11] The spring constant of a structure is proportional to its cross section area divided by its length and hence spring constant decreases with size.

[12] V. S. Ilchenko and M. L. Gorodetsky, Laser Phys. 2, 1004 (1992).

[13] D. K. Armani et al., Nature (London) 421, 925 (2003).

[14] S. M. Spillane et al., Phys. Rev. Lett. 91, 043902 (2003).

[15] M. Cai, O. Painter, and K. J. Vahala, Phys. Rev. Lett. 85, 74 (2000).

[16] M. L. Gorodetsky and V. S. Ilchenko, J. Opt. Soc. Am. B 16, 147 (1999).

[17] This view is approximate, however, and cannot replace the more accurate Eqs. (1)-(3).

[18] Analytic equations for thresholds will be shown elsewhere, T. J. Kippenberg, H. Rokhsari, T. Carmon, A. Scherer, and K. J. Vahala, Phys. Rev. Lett. (to be published).

[19] Since the lifetime of the excited state is short enough for it to decay when circulating pump power in cavity drops [as shown in Fig. 3(e)]. 